\title{
Correlation between histological invasiveness and the computed tomography value in pure ground-glass nodules
}

\author{
Akihiko Kitamii $^{1} \cdot$ Fumitoshi Sano $^{1} \cdot$ Shoko Hayashi $^{1} \cdot$ Kosuke Suzuki $^{1}$ • \\ Shugo Uematsu ${ }^{1} \cdot$ Yoshito Kamio $^{1} \cdot$ Takashi Suzuki $^{1} \cdot$ Mitsutaka Kadokura $^{3}$. \\ Mutsuko Omatsu ${ }^{2} \cdot$ Toshiaki Kunimura ${ }^{2}$
}

Received: 17 March 2015 / Accepted: 8 June 2015 / Published online: 30 June 2015

(C) The Author(s) 2015. This article is published with open access at Springerlink.com

\begin{abstract}
Purpose The purpose of this study was to evaluate the correlation between histological invasiveness and the computed tomography (CT) value and size in pure ground-glass nodules (GGNs) to determine optimal "follow-up or resection" strategies.

Methods Between 2001 and 2014, 78 resected, pure GGNs were retrospectively evaluated. The maximum diameter and CT value of pure GGNs were measured using a computer graphics support system.

Results All GGNs with a maximum diameter $\leq 10 \mathrm{~mm}$ and CT value $\leq-600$ Hounsfield units (HU) were considered to be noninvasive lesions, while 21 of $26(81 \%)$ with a maximum diameter $>10 \mathrm{~mm}$ and $\mathrm{CT}$ value $>-600 \mathrm{HU}$ were considered to be invasive lesions. With respect to the correlation between each histological type and pure GGN with a maximum diameter $\leq 10 \mathrm{~mm}$ and $\mathrm{CT}$ value $\leq-600$ $\mathrm{HU}$, the specificity was $90 \%$ and the sensitivity and negative predictive value were both $100 \%$ in atypical adenomatous hyperplasia (AAH), while the specificity was $58 \%$ and the sensitivity and positive predictive value were $0 \%$ in minimally invasive and invasive adenocarcinoma.

Conclusion Pure GGNs with a maximum diameter of $\leq 10 \mathrm{~mm}$ and CT value of $\leq-600 \mathrm{HU}$ are nearly always

Akihiko Kitami

akitami@med.showa-u.ac.jp

1 Respiratory Disease Center, Showa University Northern Yokohama Hospital, 35-1 Chigasaki-Chuo, Tsuzuki-ku, Yokohama-City 224-8503, Japan

2 Department of Clinical Diagnostic Pathology, Showa University Northern Yokohama Hospital, Yokohama, Japan

3 Division of Chest Surgery, Showa University School of Medicine, Tokyo, Japan
\end{abstract}

pre-invasive lesions; therefore, surgery should be carefully selected in such patients.

Keywords Lung cancer $\cdot$ Diagnosis $\cdot$ Surgery $\cdot$ Computed tomography

\section{Introduction}

A ground-glass opacity (GGO) is defined as a shadow completely occupied by a hazy increased attenuation of the lung, with reservation of the bronchial and vascular margins in the lesion on HRCT. Among nodules detected on computed tomography (CT), a substantial number have GGO and are therefore referred to as ground-glass nodules (GGNs). Nonsolid GGNs (pure GGNs) are homogeneous lesions; however, they exhibit variations in the CT findings with respect to value and size. The histological features of persistent pure GGNs include atypical adenomatous hyperplasia (AAH), adenocarcinoma in situ (AIS), minimally invasive adenocarcinoma (MIA), and occasionally invasive adenocarcinoma (Ad.) such as lepidic predominant adenocarcinoma (LPA). The purpose of this study was to evaluate the correlation between histological invasiveness and the CT value and size in pure GGNs to determine optimal "follow-up or resection" strategies for lesions displaying pure GGNs.

\section{Patients and methods}

We recommended resection of GGN lesions that did not decrease in size during 3 months of follow-up prior to 2009. Since 2010, we defined GGN lesions with a maximum diameter $>10 \mathrm{~mm}$ and $\mathrm{CT}$ value $>-600 \mathrm{HU}$ [1]. 


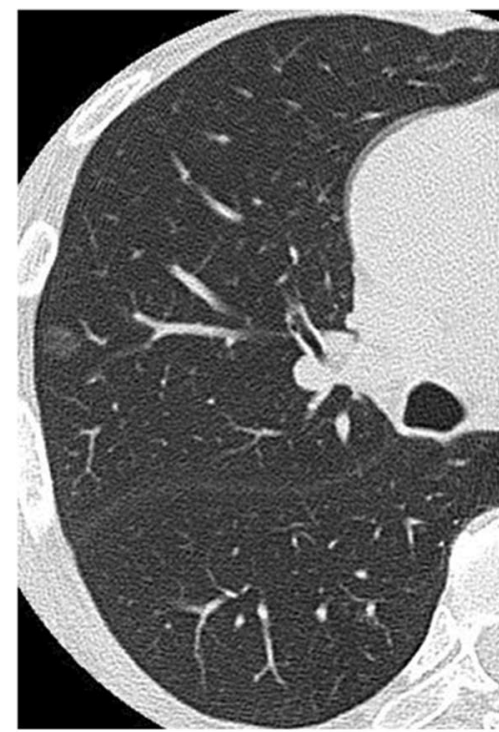

$9 \mathrm{~mm},-760 \mathrm{HU}$

Atypical adenomatous hyperplasia

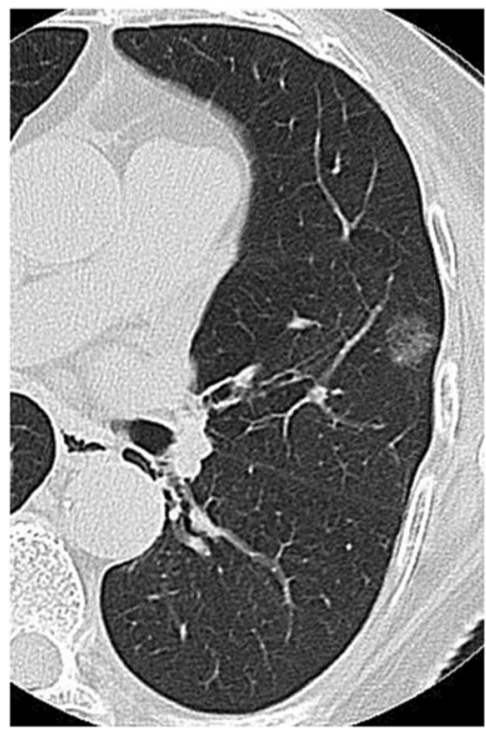

$12 \mathrm{~mm},-630 \mathrm{HU}$

Adenocarcinoma in situ

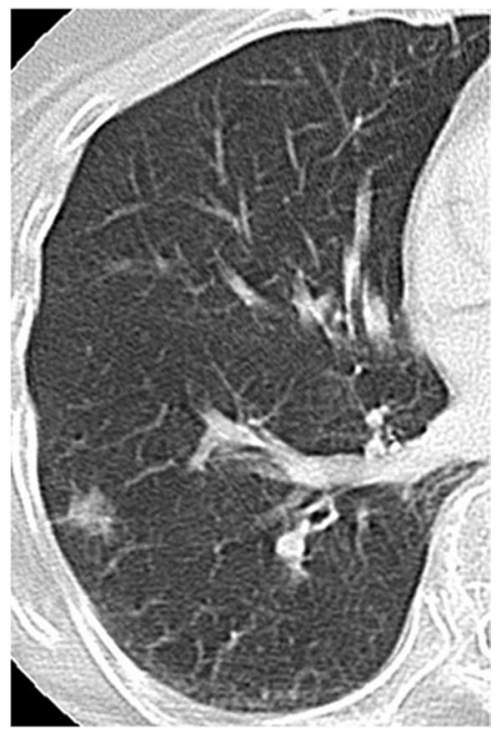

$10 \mathrm{~mm},-311 \mathrm{HU}$

Lepidic predomonant adenocarcinoma

Fig. 1 Radiologic findings of pure GGN

Between April 2001 and September 2014, a total of 84 pure GGNs in 81 patients were resected at our hospital. Of these, 78 nodules in 72 patients with pure GGNs $\leq 30 \mathrm{~mm}$ in size were evaluated in this retrospective analysis. Five patients had multiple pure GGNs, and three patients had pure GGNs and solid or partially solid adenocarcinomas.

During this period, 45 pure GGNي in 39 patients were followed up using HRCT at 4-6 months interval. Forty-one GGNs were lesions with a maximum diameter $\leq 10 \mathrm{~mm}$ or CT value $\leq-600 \mathrm{HU}$, and four GGNs were lesions with a maximum diameter $>10 \mathrm{~mm}$ and $\mathrm{CT}$ value $>-600 \mathrm{HU}$.

Non-enhanced CT scans were performed from lung apices to bases during breath-holding at mid-inspiration using a CT scanner (Aquilion 64, Toshiba, Tokyo, Japan). The helical scanning protocol was as follows: $120 \mathrm{kVp} ; 200 \mathrm{~mA}$; 0.5-s scanning time; window level, -500 Hounsfield units (HU); window width, $1600 \mathrm{HU}$; and a $512 \times 512$ matrix corresponding to a pixel size of approximately $0.6 \mathrm{~mm}$. HRCT images were reconstructed at $0.5-\mathrm{mm}$ intervals with a high-spiral-frequency algorithm (bone algorithm). Pure GGN was defined as a shadow completely occupied by a hazy increased attenuation of the lung, with reservation of the bronchial and vascular margins in the lesion with no solid regions on HRCT. The maximum diameter and $\mathrm{CT}$ value were measured using a computer graphics support system (HOPE/DrABLE-EX. Fujitsu, Tokyo, Japan). The CT value of each GGN lesion was measured in two or three areas excluding portions of apparent vessels, and the highest value in nonhomogeneous lesions was selected. Histological diagnoses were based on the new classification of lung adenocarcinoma proposed by the International Association for the Study of Lung Cancer, the American Thoracic Society, and the European Respiratory Society and classified as adenocarcinoma in situ (AIS), minimally invasive adenocarcinoma (MIA), and invasive adenocarcinoma (Ad.) [lepidic predominant adenocarcinoma (LPA) and other histological subtypes] [2]. We classified AAH and AIS as pre-invasive lesions, and MIA and Ad. as invasive lesions. Radiologic findings of three representative cases are shown in Fig. 1.

Statistical analyses were performed using SPSS II (SPSS Inc; Chicago, IL, USA). All data were expressed as the mean \pm standard deviation (SD). The differences in mean and SD values were analyzed between groups using a two-tailed Student's $t$ test. Between-group differences with $p$ values less than 0.05 were considered to be statistically significant.

\section{Results}

Patients ranged in age from 39 to 83 years (median, 64 years). Forty patients were female and 32 were male. The maximum tumor dimension was $\leq 10 \mathrm{~mm}$ in 30 nodules, $11-20 \mathrm{~mm}$ in 36 nodules, and $21-30 \mathrm{~mm}$ in 11 nodules. The mean maximum tumor dimension of all cases was $12.9 \pm 6.1 \mathrm{~mm}$. The mean CT value of all cases was $-569 \pm 126 \mathrm{HU}$. 


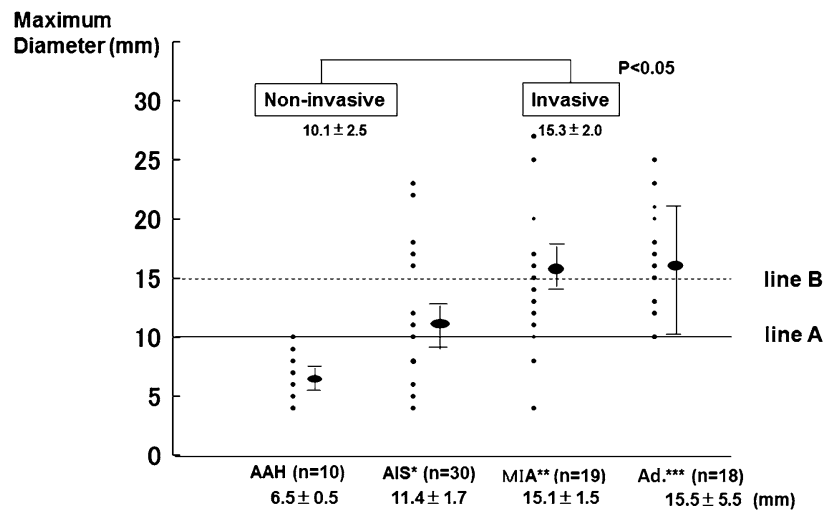

Fig. 2 The plots of the maximum diameter in AAH, AIS, MIA, and Ad. The percentage of invasive lesions was $19 \%(6 / 31)$ for lesions $\leq 10 \mathrm{~mm}$ (line A), while it was $38 \%$ (20/53) for lesions $\leq 15 \mathrm{~mm}$ (line $B)$. The difference between the noninvasive and invasive lesions was significant $(p<0.05)$. Some plots with the same size overlap at the same point. Bars mean $\pm \mathrm{SD}$. AIS* adenocarcinoma in situ, MIA** minimally invasive adenocarcinoma, $A d$.*** invasive adenocarcinoma

The mean interval from initial detection of focal GGO to pulmonary resection was 18 months (range 3-130 months). Pathologic specimens demonstrated AAH in 10 nodules, AIS in 30 nodules, MIA in 19 nodules, and Ad. in 18 nodules, including 14 cases of LPA and lymphoproliferative disorder in 1 nodule. Surgical procedures included lobectomy in 30 nodules, segmentectomy in 15 nodules, and partial resection in 32 nodules. None of the 47 patients, except for 1 with solid adenocarcinoma who underwent lymph node dissection and sampling, had lymph node involvement. All patients, except for two who died from other diseases and three who experienced recurrence accompanied with solid adenocarcinoma, have survived with no evidence of tumor recurrence to date, at a median follow-up of 46 months. Figure 2 shows the distribution of the maximum diameter in AAH, AIS, MIA, and Ad. The mean maximum diameter in AAH, AIS, MIA, and Ad was $6.5 \pm 0.5 \mathrm{~mm}, 11.4 \pm 1.7 \mathrm{~mm}, 15.1 \pm 1.5 \mathrm{~mm}$, and $15.1 \pm 5.5 \mathrm{~mm}$, respectively. The percentage of invasive lesions was $19 \%(6 / 31)$ among lesions $\leq 10 \mathrm{~mm}$ (line A) and $38 \%(20 / 53)$ in lesions $\leq 15 \mathrm{~mm}$ (line B). The difference between the noninvasive and invasive lesions was statistically significant $(p<0.05)$. Figure 3 shows the distribution of the CT values in AAH, AIS, MIA, and Ad. The mean CT value in AAH, AIS, MIA, and Ad was $-699 \pm 23 \mathrm{HU},-580 \pm 120 \mathrm{HU},-509 \pm 98 \mathrm{HU}$, and $-532 \pm 37 \mathrm{HU}$. All AAH lesions were less than $-600 \mathrm{HU}$ and had significantly lower CT values than AIS, MIA, and Ad. The differences between AIS and MIA or between the noninvasive and invasive lesions were statistically significant $(p<0.05)$. No significant difference in the CT values was observed between MIA and Ad. Figure 4 illustrates the relationship between the $\mathrm{CT}$ value and maximum

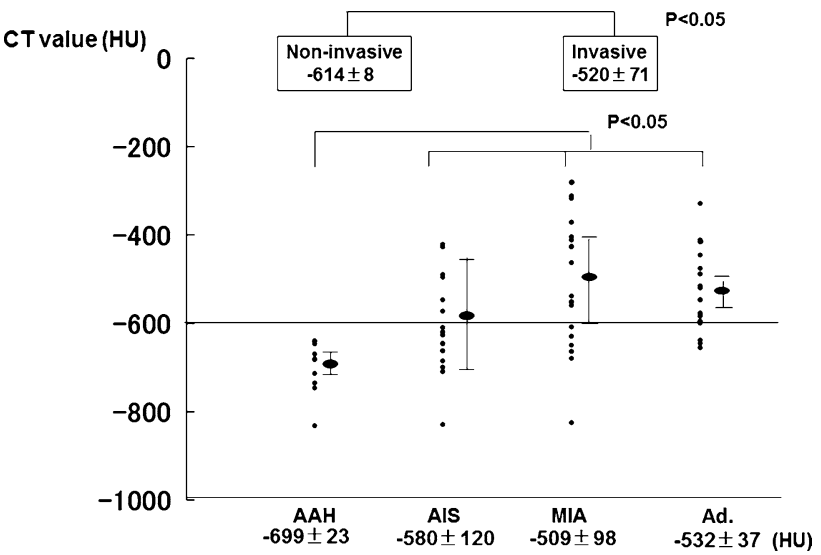

Fig. 3 CT values in AAH, AIS, MIA, and Ad. All AAH lesions were $\leq-600 \mathrm{HU}$ and had significantly lower CT values than AIS, MIA, and Ad. The differences between AIS and MIA and between the noninvasive and invasive lesions were significant $(p<0.05)$. No significant difference in the CT values was observed between MIA and Ad. Some plots with the same CT value overlap at the same point. Bars mean $\pm \mathrm{SD}$

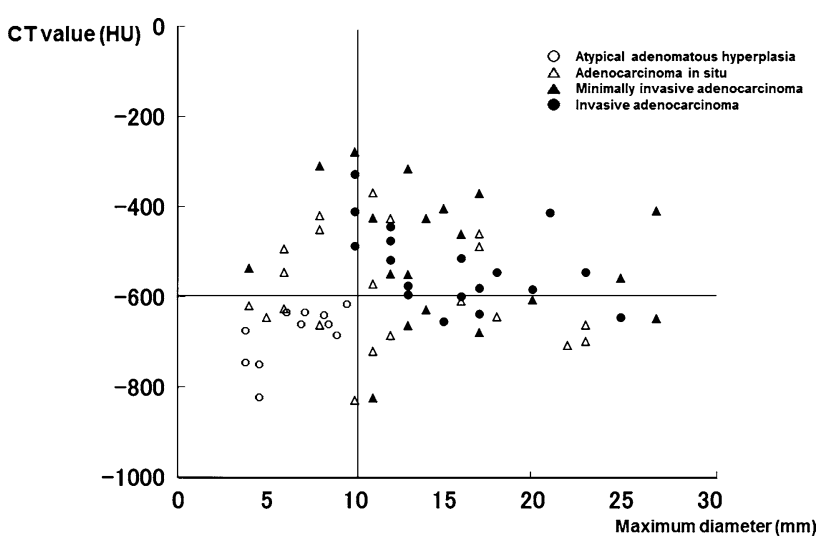

Fig. 4 Relationship between the CT value and maximum diameter of pure GGN lesions. All GGN lesions with a maximum diameter $\leq 10 \mathrm{~mm}$ and $\mathrm{CT}$ value $\leq-600 \mathrm{HU}$ were noninvasive lesions, while 21 of $26(81 \%)$ GGN lesions with a maximum diameter $>10 \mathrm{~mm}$ and $\mathrm{CT}$ value $>-600 \mathrm{HU}$ were invasive lesions. Some plots with the same size and CT value overlap at the same point

diameter of pure GGN lesions. All GGN lesions with a maximum diameter $\leq 10 \mathrm{~mm}$ and $\mathrm{CT}$ value $\leq-600 \mathrm{HU}$ were considered to be noninvasive lesions, while 21 of $26(81 \%)$ with a maximum diameter $>10 \mathrm{~mm}$ and CT value $>-600 \mathrm{HU}$ were considered to be invasive lesions. With respect to the correlation between each histological type and pure GGN lesions with a maximum diameter $\leq 10 \mathrm{~mm}$ and $\mathrm{CT}$ value $\leq-600 \mathrm{HU}$, the specificity was $90 \%$ and the sensitivity and negative predictive values were $100 \%$ in $\mathrm{AAH}$, while the specificity was $58 \%$, and the sensitivity and positive predictive values were $0 \%$ in MIA and Ad (Table 1). 
Table 1 Correlation of histology and pure GGN lesions with a maximum diameter $\leq 10 \mathrm{~mm}$ and CT value $\leq-600 \mathrm{HU}$

\begin{tabular}{|c|c|c|c|c|c|}
\hline $\mathrm{AAH}$ & AAH & nonAAH & Total & & \\
\hline$\leq-600 \mathrm{HU}$ and $\leq 10 \mathrm{~mm}$ & 10 & 7 & 17 & Specificity & $90 \%$ \\
\hline$>-600 \mathrm{HU}$ or $>10 \mathrm{~mm}$ & 0 & 60 & 60 & Sensitivity & $100 \%$ \\
\hline \multirow[t]{2}{*}{ Total } & 10 & 67 & 77 & Positive predictive value & $59 \%$ \\
\hline & & & & Negative predictive value & $100 \%$ \\
\hline AIS & AIS & non AIS & Total & & \\
\hline$\leq-600 \mathrm{HU}$ and $\leq 10 \mathrm{~mm}$ & 7 & 10 & 17 & Specificity & $76 \%$ \\
\hline$>-600 \mathrm{HU}$ or $>10 \mathrm{~mm}$ & 23 & 28 & 60 & Sensitivity & $23 \%$ \\
\hline \multirow[t]{2}{*}{ Total } & 30 & 37 & 77 & Positive predictive value & $41 \%$ \\
\hline & & & & Negative predictive value & $47 \%$ \\
\hline MIA + Ad. & MIA + Ad. & Others & Total & & \\
\hline$\leq-600 \mathrm{HU}$ and $\leq 10 \mathrm{~mm}$ & 0 & 17 & 17 & Specificity & $58 \%$ \\
\hline$>-600 \mathrm{HU}$ or $>10 \mathrm{~mm}$ & 37 & 23 & 60 & Sensitivity & $0 \%$ \\
\hline \multirow[t]{2}{*}{ Total } & 37 & 40 & 77 & Positive predictive value & $0 \%$ \\
\hline & & & & Negative predictive value & $38 \%$ \\
\hline
\end{tabular}

As concerns AAH, specificity is $90 \%$, sensitivity and negative predictive value are $100 \%$. While, in MIA and invasive adenocarcinoma, specificity is $58 \%$, sensitivity and positive predictive value are $0 \%$

In 45 follow-up cases of GGNs, two $(50 \%)$ of four lesions with a maximum diameter $>10 \mathrm{~mm}$ and $\mathrm{CT}$ value $>-600 \mathrm{HU}$ and $11(24 \%)$ of 41 lesions $\leq 10 \mathrm{~mm}$ or CT value $\leq-600 \mathrm{HU}$ had findings of increased size or CT number. The median follow-up period was 51 months.

\section{Discussion}

Many radiologic studies of small lung adenocarcinomas have demonstrated a strong correlation between the CT findings and pathologic features [3, 4]. Several groups have classified small lung lesions into the nonsolid (pure) GGO type, partly solid (mixed) GGO type, and solid type and have suggested that the pure type and mixed type with small solid components are nearly always noninvasive carcinomas [5-8]. However, it is occasionally difficult to differentiate between pure and mixed GGO and between high-density GGO and a solid tumor. Suzuki et al. classified homogeneous (so-called "pure") GGO into pure GGO and semiconsolidation to evaluate the differences in the density within the tumor and determined that, pathologically, semiconsolidation tends to be adenocarcinoma with invasive foci [9]. Although the differentiation between pure GGN and semiconsolidation was unclear in their report, the density within homogeneous GGNs appears to be an important factor for predicting tumor invasiveness. We speculated that pure GGNs should be defined as homogeneous hazy lesions, for which CT values were $\leq-300 \mathrm{HU}$ in the present analysis. Because $-300 \mathrm{HU}$ is the limitation of differentiation between vessels, we classified lesions with $\mathrm{CT}$ values $>-300 \mathrm{HU}$ to be solid nodules.

Several groups have used quantitative densitometric methodologies to evaluate GGN lesions [10-13]. Ikeda et al. reported that the 75th percentile CT value analyzed using three-dimensional computerized quantification of the GGO lesions was the optimal value for differentiating between AAH, BAC, and adenocarcinoma. These investigators noted that a CT cutoff value of $-584 \mathrm{HU}$ was optimal for differentiating $\mathrm{AAH}$ and $\mathrm{BAC}$, and $-472 \mathrm{HU}$ was optimal for differentiating BAC and adenocarcinoma [11]. Nomori et al. used histograms of CT pixel numbers for $\mathrm{AAH}$ and nonmucinous adenocarcinoma to quantify the peaks and mean numbers of CT pixels and found the mean CT values to be $-697 \pm 56 \mathrm{HU}$ for AAH lesions and $-541 \pm 73 \mathrm{HU}$ for BAC lesions. These investigators noted that the peak CT value on the histogram was the most frequent value observed in the tumor and that the effect of vessels and bronchi within the tumor can be ignored [12] The CT value of each GGN lesion was measured in two or three areas, excluding the apparent vessels, in this study, and was the selected highest value in nonhomogeneous lesions. The present study demonstrates that a CT value of $-600 \mathrm{HU}$ may represent a cutoff value demarcating AAH versus invasive lesions. We believe that this finding could significantly impact the selection of therapeutic strategies for GGN lesions.

Whether GGN lesions that are suspected to be preinvasive or minimally invasive adenocarcinoma should be resected or followed up also remains controversial. Recently, several groups have reported that for pre-invasive 
lesions identified on HRCT, careful observation without surgical intervention represents a valid treatment option [14-16]. According to the interim guidelines suggested by Godoy and Nadich, isolated lesions with pure GGNs that are $\leq 5 \mathrm{~mm}$ do not necessarily require CT follow-up, since they nearly always represent foci of AAH; however, for lesions between 5 and $10 \mathrm{~mm}$, follow-up is mandatory pending better definition of their true nature. Lesions $>1 \mathrm{~cm}$ should be assumed to be so-called BAC or invasive adenocarcinoma; however surgery should be considered, particularly if the nodule is growing or if an increase in attenuation or development of a solid component is observed [17].

Diligent long-term follow-up of the natural history of pure GGN and should be conducted to determine whether surgical intervention is acceptable or unnecessary [18]. However, we believe that early detection and early therapy of some primary lung cancers with pure GGNs are important for improving individual prognoses for the following reasons. First, pure GGN lesions are not all histologically pre-invasive, as some, such as MIA and LPA, have an Ad. component. Second, several groups have reported the concept of a multi-step progression from AAH through localized BAC (AIS and MIA) to advanced adenocarcinoma with a replacement growth pattern (LPA and other type of Ad.) [19-21]. Third, patients with LPA have a lower 5-year survival rate (approximately $93 \%$ ) than patients with AIS and MIA (100\%) [22-25]. Our therapeutic strategy for pure GGNs is to avoid unnecessary surgery for AAH and therapeutic delays for Ad.

There are some limitations associated with this study, such as a retrospective study design and small number of surgical cases. However, we revealed a correlation between the histology and pure GGN lesions with a maximum diameter $\leq 10 \mathrm{~mm}$ and $\mathrm{CT}$ value $\leq-600 \mathrm{HU}$. With respect to AAH, the specificity was $90 \%$ and the sensitivity and negative predictive values were both $100 \%$. In contrast to MIA and Ad., the specificity was $58 \%$ and the sensitivity and positive predictive values were both $0 \%$. According to these results, we propose the follow-up criteria of a maximum diameter $\leq 10 \mathrm{~mm}$ and $\mathrm{CT}$ value $\leq-600 \mathrm{HU}$ for pure GGNs, and unnecessary surgery for AAH and therapeutic delay for Ad. can be avoided.

\section{Conclusion}

Pure GGNs at a maximum diameter of $\leq 10 \mathrm{~mm}$ and CT value of $\leq-600 \mathrm{HU}$ are nearly always pre-invasive lesions; therefore, surgery should be carefully selected in such patients.

Conflict of interest The authors declare that they have no conflicts of interest.
Open Access This article is distributed under the terms of the Creative Commons Attribution 4.0 International License (http://creativecommons.org/licenses/by/4.0/), which permits unrestricted use, distribution, and reproduction in any medium, provided you give appropriate credit to the original author(s) and the source, provide a link to the Creative Commons license, and indicate if changes were made.

\section{References}

1. Kitami A, Kamio Y, Hayashi S, Suzuki K, Uematsu S, Gen R, et al. One-dimensional mean computed tomography value evaluation of ground-glass opacity on high-resolution images. Gen Thorac Cardiovasc Surg. 2012;60:425-30.

2. Travis WD, Brambilla E, Noguchi M, Nicholson AG, Geisinger $\mathrm{KR}$, Yatabe $\mathrm{Y}$, et al. International association for the study of lung cancer/american thoracic society/european respiratory society international multidisciplinary classification of lung adenocarcinoma. J Thorac Oncol. 2011;6:244-85.

3. Imai K, Minamiya Y, Saito H, Motoyama S, Sato Y, Ito A, et al. Diagnostic imaging in the preoperative management of lung cancer. Surg Today. 2014;44:1197-206.

4. Hattori A, Suzuki K, Takamochi K, Oh S. Clinical features of multiple lung cancers based on thin-section computed tomography: what are the appropriate surgical strategies for second lung cancers? Surg Today. 2015;45:189-96.

5. Kodama K, Higashiyama M, Yokouchi H, Takami K, Kuriyama $\mathrm{K}$, Mano $\mathrm{M}$, et al. Prognostic value of ground-glass opacity found in small lung adenocarcinoma on high-resolution CT scanning. Lung Cancer. 2001;33:17-25.

6. Takashima S, Maruyama Y, Hasegawa M, Yamanda T, Honda T, Kadoya M, et al. Prognostic significance of high-resolution CT findings in small peripheral adenocarcinoma of the lung: a retrospective study on 64 patients. Lung Cancer. 2002;36:289-95.

7. Nakata M, Saeki H, Takata I, Segawa Y, Mogami H, Mandai K, et al. Focal ground-glass opacity detected by low-dose helical CT. Chest. 2002;121:1464-7.

8. Matsuguma H, Nakahara R, Anraku M, Kondo T, Tsuura Y, Kamiyama $\mathrm{Y}$, et al. Objective definition and measurement method of ground-glass opacity for planning limited resection in patients with clinical stage IA adenocarcinoma of the lung. Eur $\mathbf{J}$ Cardiothorac Surg. 2004;25:1102-6.

9. Suzuki K, Kusumoto M, Watanabe S, Tsuchiya R, Asamura H. Radiologic classification of small adenocarcinoma of the lung: radiologic-pathologic correlation and its prognostic impact. Ann Thorac Surg. 2006;81:413-20.

10. Yang ZG, Sone S, Takashima S, Li F, Honda T, Maruyama $\mathrm{Y}$, et al. High-resolution $\mathrm{CT}$ analysis of small peripheral lung adenocarcinoma revealed on screening helical CT. AJR. 2001;176:1339-407.

11. Ikeda K, Awai K, Mori T, Kawanaka K, Yamashita Y, Nomori H. Differential diagnosis of ground-glass opacity nodules. CT number analysis by three dimensional computerized quantification. Chest. 2007;132:984-90.

12. Nomori H, Ohtsuka T, Naruke T, Suemasu K. Differentiating between atypical adenomatous hyperplasia and bronchioloalveolar carcinoma using the computed tomography number histogram. Ann Thorac Surg. 2003;76:867-71.

13. Yanagawa M, Kuriyama K, Kunitomi Y, Tomiyama N, Honda O, Sumikawa H. One-dimensional quantitative evaluation of peripheral lung adenocarcinoma with or without ground-glass opacity on thin-section CT images using profile curve. Br J Radiol. 2009;82:532-40. 
14. Suzuki K, Asamura H, Kusumoto M, Kondo H, Tsuchiya R. "Early" peripheral lung cancer: prognostic significance of ground glass opacity on thin-section computed tomographic scan. Ann Thorac Surg. 2002;74:1635-9.

15. Kakinuma R, Ohmatsu H, Kaneko M, Kusumoto M, Yoshida J, Nagai K, et al. Progression of focal pure ground-glass opacity detected by low-dose helical computed tomography screening for lung cancer. J Conmut Assist Tomogr. 2004;28:17-23.

16. Asamura H, Suzuki K, Watanabe S, Matsuno Y, Maeshima A, Tsuchiya R. A clinicopathological study of resected subcentimeter lung cancers: a favorable prognosis for ground glass opacity lesions. Ann Thorac Surg. 2003;76:1016-22.

17. Godoy MCB, Nadich DP. Subsolid pulmonary nodules and the spectrum of peripheral adenocarcinoma of the lung: recommended interim guidelines for assessment and management. Radiology. 2009;253:606-22.

18. Kodama K, Higashiyama M, Yokouchi H, Takami K, Kuriyama K, Kusunoki Y, et al. Natural history of pure ground-glass opacity after long-term follow-up of more than 2 years. Ann Thorac Surg. 2002;73:386-93.

19. Noguchi M, Shimosato Y. The development and progression of adenocarcinoma of the lung. Cancer Treat Res. 1995;72:131-42.

20. Kitamura H, Kameda Y, Nakamura M, Nakatani Y, Inayama Y, Iida $\mathrm{M}$, et al. Proliferative potential and p53 overexpression in precursor and early stage lesions of bronchioloalveolar lung carcinoma. Am J Pathol. 1995;146:876-87.

21. Takashima S, Maruyama Y, Hasegawa M, Yamada T, Honda T, Kadoya M, et al. CT findings and progression of small peripheral lung neoplasms having a replacement growth pattern. AJR. 2003;180:817-26.

22. Russell PA, Wainer Z, Wright GM, Daniels M, Conron M, Williams RA. Does lung adenocarcinoma subtype predict patient survival? A clinicopathologic study based on the new International Association for the Study of Lung Cancer/American Thoracic Society/European Respiratory Society international multidisciplinary lung adenocarcinoma classification. J Thorac Oncol. 2011;6:1496-504.

23. Yoshizawa A, Sumiyoshi S, Sonobe M, Kobayashi M, Fujimoto M, Kawakami F, Tsuruyama T, Travis WD, Date H, Haga H. Validation of the IASLC/ATS/ESR lung adenocarcinoma classification for prognosis and association with EGFR and KRAS gene mutations: analysis of 440 Japanese patients. J Thorac Oncol. 2013;8:52-61.

24. Tsuta K, Kawago M, Inoue E, Yoshida A, Takahashi F, Sakurai H, Watanabe S, Takeuchi M, Furuta K, Asamura H, Tsuda H. The utility of the proposal IASLC/ATS/ERS lung adenocarcinoma subtype for disease prognosis and correlation of driver gene alteration. Lung Cancer. 2013;81:371-6.

25. Kadota K, Villena-Vargas J, Yoshizawa A, Motoi N, Sima CS, Riely GJ, Rusch VW, Adusumilli PS, Travis WD. Prognostic significance of adenocarcinoma in situ, minimally invasive adenocarcinoma, and nonmucinous lepidic predominant invasive adenocarcinoma of the lung in patients with stage I disease. Am J Surg Pathol. 2014;38:448-60. 